\title{
Theta - Vacuum of the Bosonized Massive Light-Front Schwinger Model
}

\author{
L’ubomír Martinovič \\ Institute of Physics, Slovak Academy of Sciences \\ Dúbravská cesta 9, 84228 Bratislava, Slovakia * \\ and \\ International Institute of Theoretical and Applied Physics, \\ Iowa State University, Ames, Iowa 50011, USA \\ James P. Vary \\ Department of Physics and Astronomy \\ and \\ International Institute of Theoretical and Applied Physics, \\ Iowa State University, Ames, Iowa 50011, USA
}

October 5, 1998

\begin{abstract}
The massive Schwinger model in bosonic representation is quantized on the light front using the Dirac-Bergmann method. The non-perturbative theta vacuum in terms of coherent states of the gauge-field zero mode is derived and found to coincide with the massless case. On the other hand, the mass term becomes highly non-linear due to the constrained zero mode of the scalar field. A non-trivial mixing between the normal-mode and zero-mode sectors of the model is crucial for the correct calculation of the theta dependence of the leading order mass correction to the chiral condensate.
\end{abstract}

\section{Introduction}

Massless quantum electrodynamics in two dimensions [1], the Schwinger model, is a solvable model with a surprising richness of non-perturbative phenomena. It has become a useful testing ground for new methods in quantum field theory including the method of discretized light-cone quantization (DLCQ) [2]. Full mass spectra with the corresponding wave functions have been obtained for broad ranges of the coupling constant (and the fermion mass). It turns out however that it is much more difficult to understand such non-perturbative aspects known from usual formulation as chiral anomaly, $\theta$ vacuum and chiral symmetry breaking within DLCQ [3]. It is even not quite clear which degrees of freedom are responsible for these phenomena.

* permanent address 
An attempt to derive a full operator solution of the light-front Schwinger model has been undertaken by McCartor [⿰亻⿱㇒士幺] (see also [5]). An important ingredient in this approach was an initialization of quantum fields on the both characteristic surfaces $x^{+}=0, x^{-}=0$. Consequently, some advantages of the light-front (LF) formulation (first of all the "triviality" of the LF vacuum) have been lost. A genuine light-front solution equivalent to that of the covariant formulation [6, [7] has not been given so far.

On the other hand, some progress in understanding non-perturbative aspects of LF field theories has been achieved by studying the constrained and dynamical zero modes of bosonic fields. They appear due to periodic boundary conditions in the finite-volume formulation of the theory [8,9]. In particular, the dynamical zero mode of the $A^{+}$gauge-field component [10] has been shown to lead to a non-trivial vacuum structure in the bosonized (massless) Schwinger model within the Schrödinger coordinate representation [11] as well as in the coherent state approach in the Fock representation [12].

In addition, improved light-front gaussian effective potential methods [14 and near light-front quantization approaches [15 have provided access to the condensate and chiral corrections.

The purpose of the present paper is to give a generalization of the coherentstate approach [12] for the case of non-vanishing mass of the fermion field. The Lagrangian of the bosonized theory is employed for simplicity. This helps to avoid so far unsolved problems with massless LF fermions in two dimensions [3, 4] while still allows to study some non-trivial aspects of the LF vacuum structure. We choose the Dirac-Bergmann quantization method to correctly handle the constraints present in both the normal and zero Fourier mode sectors. The procedure generates a secondary constraint which relates the zero mode of the scalar field and the gauge field conjugate momentum. As a consequence, the bosonized fermion mass term becomes a complicated non-linear function of zero-mode variables. This can be understood as the price of constructing the exact ground state of the theory.

Based on the quantum realization of the residual symmetry of the gauge zero mode under constant shifts, the true physical (gauge invariant) vacuum is indeed derived in terms of coherent states of the dynamical gauge field. The validity of the theta-vacuum construction is demonstrated by a calculation of the lowest order fermion-mass correction to the chiral condensate. The correct treatment of the commutators between the zero and normal modes of the scalar field plays a crucial role here.

\section{Bosonized massive LF Schwinger model in a finite box}

Aside from a variational treatment [16], the only known method to study properties of the vacuum state of two-dimensional LF field theories is to restrict the system to a finite interval $-L \leq x^{-} \leq L$. This can be viewed as a convenient infrared regularization which facilitates disentangling the vacuum aspects $\left(k^{+}=0\right.$ modes of quantum fields) from the remainder of the dynamics. The 
decomposition of the fields into the zero-mode (ZM, subscript 0) and normalmode (NM, subscript $\mathrm{n}$ ) parts can easily be performed by imposing periodic boundary conditions on the corresponding fields 7 . For example, the gauge and scalar fields, whose dynamics will be studied below, are decomposed as (our convention is $x^{ \pm}=x^{0} \pm x^{1}$ )

$$
A^{\mu}\left(x^{+}, x^{-}\right)=A_{0}^{\mu}\left(x^{+}\right)+A_{n}^{\mu}\left(x^{+}, x^{-}\right), \phi\left(x^{+}, x^{-}\right)=\phi_{0}\left(x^{+}\right)+\phi_{n}\left(x^{+}, x^{-}\right) .
$$

Before proceeding with the Dirac-Bergmann (DB) quantization procedure, let us recall the relation between the fermionic and bosonic formulations of the massive Schwinger model. The Lagrangian density of the two-dimensional spinor field $\psi$ of mass $\mathrm{m}$ interacting with the gauge field $A^{\mu}$ is

$$
\mathcal{L}=-\frac{1}{4} F^{\mu \nu} F_{\mu \nu}+\bar{\psi} i \gamma^{\mu} D_{\mu} \psi-m \bar{\psi} \psi
$$

In the light-front formulation with the finite-volume light cone gauge $A_{n}^{+}=$ $0, A_{0}^{-}=0$ we have

$$
\begin{aligned}
\mathcal{L}_{f} & =2 i \psi_{+}^{\dagger} \partial_{+} \psi_{+}+2 i \psi_{-}^{\dagger} \partial_{-} \psi_{-}+\frac{1}{2}\left(\partial_{+} A_{0}^{+}\right)^{2}+\frac{1}{2}\left(\partial_{-} A_{n}^{-}\right)^{2} \\
& -m\left(\psi_{+}^{\dagger} \psi_{-}+\psi_{-}^{\dagger} \psi_{+}\right)-\frac{e}{2} j_{n}^{+} A_{n}^{-}-\frac{e}{2} j_{0}^{-} A_{0}^{+} .
\end{aligned}
$$

Here $\psi_{+}$and $\psi_{-}$are respectively the dynamical and dependent fermi-field components

$$
\psi_{+}=\Lambda_{+} \psi, \psi_{-}=\Lambda_{-} \psi, \Lambda_{ \pm}=\frac{1}{2} \gamma^{0} \gamma^{ \pm}, \gamma^{ \pm}=\gamma^{0} \pm \gamma^{1}
$$

The bosonized form of the theory is obtained by the correspondences 18, 19

$$
\begin{gathered}
i \bar{\psi} \gamma^{\mu} \partial_{\mu} \psi=\frac{1}{2} \partial^{\mu} \phi \partial_{\mu} \phi, \\
j^{\mu}=\frac{1}{\sqrt{\pi}} \epsilon^{\mu \nu} \partial_{\nu} \phi \\
\bar{\psi} \psi=K: \cos c \phi:
\end{gathered}
$$

where $\phi(x)$ is the equivalent real boson field. The other symbols in (5) - (7) are

$$
K=\frac{\mu}{2 \pi} e^{\gamma_{E}}, \quad \mu=\frac{e}{\sqrt{\pi}}, \quad \epsilon^{\mu \nu}=-\epsilon^{\nu \mu}, \quad c=2 \sqrt{\pi} ;
$$

$\mu$ is the Schwinger boson mass and $\gamma_{E}$ is Euler's constant. The bosonized Lagrangian density in the chosen gauge has the form

$$
\begin{aligned}
\mathcal{L}_{b} & =2 \partial_{+} \phi_{n} \partial_{-} \phi_{n}+\frac{1}{2}\left(\partial_{+} A_{0}^{+}\right)^{2}+\frac{1}{2}\left(\partial_{-} A_{n}^{-}\right)^{2}-\mu A_{n}^{-} \partial_{-} \phi_{n} \\
& +\mu A_{0}^{+} \partial_{+} \phi_{0}-m K: \cos \left(c \phi_{n}+c \phi_{0}\right): .
\end{aligned}
$$

Note that one had to fix the gauge already in the fermionic Lagrangian (3) [18], because the equivalent form of the fermion kinetic energy is (trivially)

\footnotetext{
${ }^{1}$ The necessity to prescribe (quasi) periodic boundary conditions for the consistency of the LF quantization even in continuum formulation has been emphasized by Steinhardt 17.
} 
gauge invariant by itself and does not compensate the gauge variation of the interacting term. In other words, it is difficult to speak about gauge symmetry in the bosonized form of the Lagrangian obtained by the covariant bosonization rules (5) - (7). The sole remnant of the original gauge freedom is the symmetry under constant shifts of $A_{0}^{+}$, which is 'visible' only in the finite-box formulation. This symmetry under "large" gauge transformations will emerge as the source of the non-trivial vacuum structure of the model.

Proceeding with the DB procedure, we compute momenta conjugate to the fields in the Lagrangian (10):

$$
\Pi_{A_{n}^{-}}=0, \quad \Pi_{\phi_{n}}=2 \partial_{-} \phi_{n}, \quad \Pi_{A_{0}^{+}}=\partial_{+} A_{0}^{+}, \quad \Pi_{\phi_{0}}=\mu A_{0}^{+} .
$$

The momenta, which contain no $x^{+}$-derivative, give rise to the $\mathrm{NM}$ and $\mathrm{ZM}$ primary constraints

$$
\varphi_{1}=\Pi_{A_{n}^{-}}, \quad \varphi_{2}=\Pi_{\phi_{n}}-2 \partial_{-} \phi_{n}, \quad \varphi_{3}=\Pi_{\phi_{0}}-\mu A_{0}^{+} .
$$

The primary Hamiltonian is

$$
P_{p}^{-}=P_{c}^{-}+L u_{3} \varphi_{3}+\int_{-L}^{+L} \frac{d x^{-}}{2}\left[u_{1}(x) \varphi_{1}(x)+u_{2}(x) \varphi_{2}(x)\right]
$$

with the canonical LF Hamiltonian $P_{c}^{-}$

$$
P_{c}^{-}=\int_{-L}^{+L} \frac{d x^{-}}{2}\left[\Pi_{A_{0}^{+}}^{2}-\left(\partial_{-} A_{n}^{-}\right)^{2}+2 \mu A_{n}^{-} \partial_{-} \phi_{n}+2 m K: \cos c\left(\phi_{0}+\phi_{n}\right):\right]
$$

derived from the Lagrangian (3) in the standard way. One has to check $x^{+}$independence of the primary constraints next. Consistency of $\varphi_{1}$ and $\varphi_{3}$ generates secondary constraints

$$
\begin{aligned}
\chi_{1} & =\partial_{-} A_{n}^{-}+\mu \phi_{n}, \\
\chi_{2} & =\Pi_{A_{0}^{+}}-\frac{m K c}{\mu}(: \sin c \phi:)_{0} .
\end{aligned}
$$

(The subscript 0 indicates the ZM projection of the expression in the parenthesis.) Consistency of $\varphi_{2}$ as well as of $\chi_{1}$ and $\chi_{2}$ yields weak equations for the Lagrange multipliers $u_{2}, u_{1}, u_{3}$, which means that this part of the procedure terminates. Since the freedom under small gauge transformations has been removed at the Lagrangian level, all five constraints (in the order $\varphi_{1}, \varphi_{2}, \chi_{1}, \varphi_{3}, \chi_{2}$ ) are second class and can be used to calculate the matrix of their Poisson brackets. Its inverse, needed for the computation of the Dirac brackets, is

$$
C^{-1}\left(x^{-}-y^{-}\right)=\left(\begin{array}{ccccc}
\frac{\mu^{2}}{4} \mathcal{G}_{3} & \mathcal{G}_{1} & \frac{\mu}{4} \mathcal{G}_{2} & C_{14}^{-1} & 0 \\
\mathcal{G}_{1} & 0 & 0 & 0 & 0 \\
-\frac{\mu}{4} \mathcal{G}_{2} & 0 & -\frac{1}{4} \mathcal{G}_{1} & C_{34}^{-1} & 0 \\
C_{41}^{-1} & 0 & C_{43}^{-1} & 0 & \frac{L}{\mu} \frac{1}{1-\alpha_{0}} \\
0 & 0 & 0 & -\frac{L}{\mu} \frac{1}{1-\alpha_{0}} & 0
\end{array}\right)
$$


where the argument of the matrix elements has been suppressed. The NM Green's functions $\mathcal{G}_{k}\left(x^{-}-y^{-}\right)$are defined by the equations $(k=1,2,3$, no summation)

$$
\partial_{-}^{k} \mathcal{G}_{k}\left(x^{-}-y^{-}\right)=\delta_{n}\left(x^{-}-y^{-}\right)
$$

where $\delta_{n}\left(x^{-}\right)$and $\mathcal{G}_{1}\left(x^{-}\right)$, etc. are the NM parts of the periodic delta function and one half of the periodic sign function $\epsilon_{n}\left(x^{-}\right)$, respectively [20]. The symbolic matrix elements in $C^{-1}$ have the following explicit form:

$$
\begin{gathered}
C_{14}^{-1}\left(x^{-}\right)=\frac{m K c^{2}}{4 \mu\left(1-\alpha_{0}\right)} \int_{-L}^{+L} \frac{d y^{-}}{2} \mathcal{G}_{2}\left(x^{-}-y^{-}\right): \cos c \phi\left(y^{-}\right): \\
C_{34}^{-1}\left(x^{-}\right)=\frac{m K c^{2}}{4 \mu^{2}\left(1-\alpha_{0}\right)} \int_{-L}^{+L} \frac{d y^{-}}{2} \mathcal{G}_{1}\left(x^{-}-y^{-}\right): \cos c \phi\left(y^{-}\right):
\end{gathered}
$$

and similarly for $C_{41}^{-1}\left(y^{-}\right), C_{43}^{-1}\left(y^{-}\right)$. The quantity $\alpha_{0}$ is the ZM projection of

$$
\alpha\left(x^{-}\right)=\frac{m K c^{2}}{\mu^{2}}: \cos c \phi\left(x^{-}\right):
$$

The above $C_{i j}^{-1}$ belong to the mixed ZM/NM sector of the matrix $C^{-1}$, while the lower right 2 by 2 submatrix corresponds to the ZM sector.

We do not quote all calculated Dirac brackets here. In the NM sector the only relevant commutator for our purpose in the quantum theory is

$$
\left[\phi_{n}\left(x^{-}\right), \phi_{n}\left(y^{-}\right)\right]=-\frac{i}{4} \frac{1}{2} \epsilon_{n}\left(x^{-}-y^{-}\right) .
$$

The rest of the commutators can be obtained by differentiation using the corresponding constraint (12) strongly. The Dirac brackets, signified by an asterisk, in the ZM sector have a more complicated structure. The non- vanishing cases are

$$
\begin{aligned}
\left\{A_{0}^{+}, \Pi_{A_{0}^{+}}\right\}^{*} & =\frac{1}{L}\left(1-\frac{1}{1-\alpha_{0}}\right), \\
\left\{\Pi_{A_{0}^{+}}, \Pi_{\phi_{0}}\right\}^{*} & =\frac{\mu}{L} \frac{\alpha_{0}}{1-\alpha_{0}}, \quad\left\{A_{0}^{+}, \quad \phi_{0}\right\}^{*}=-\frac{1}{L \mu} \frac{1}{1-\alpha_{0}},
\end{aligned}
$$

These can be simplified by defining new variables

$$
\Pi_{0}^{-}=\Pi_{A_{0}^{+}}-\mu \phi_{0}, \quad \Pi_{0}=\Pi_{\phi_{0}}-\mu A_{0}^{+} .
$$

The only non-zero commutators in quantum theory then read

$$
\left[A_{0}^{+}, \Pi_{0}^{-}\right]=\frac{i}{L}, \quad\left[A_{0}^{+}, \phi_{0}\right]=-\frac{i}{\mu L} \frac{1}{1-\alpha_{0}} .
$$

Note, that in the massless limit $m=0, \Pi_{0}^{-}$becomes $-\mu \phi_{0}$ and both commutators coincide in agreement with direct $m=0$ calculations [11, 12]. Finally, there is one independent non-zero mixed commutator

$$
\left[\phi_{n}\left(x^{-}\right), \phi_{0}\right]=-i \frac{m K c^{2}}{4 \mu L\left(1-\alpha_{0}\right)} \int_{-L}^{+L} \frac{d y^{-}}{2} \mathcal{G}_{1}\left(x^{-}-y^{-}\right): \cos c \phi\left(y^{-}\right):
$$


The quantum LF Hamiltonian, which depends only on unconstrained field variables, is obtained by inserting the constraints $\chi_{1}$ and $\chi_{2}$ into the primary Hamiltonian (14) as strong operator relations:

$$
P^{-}=\left[\frac{m K c}{\mu}(: \sin c \phi:)_{0}\right]^{2}+\int_{-L}^{+L} \frac{d x^{-}}{2}\left[\mu^{2} \phi_{n}^{2}\left(x^{-}\right)+2 m K: \cos c \phi\left(x^{-}\right):\right] .
$$

This completes the LF Hamiltonian quantization of the massive Schwinger model.

\section{Residual gauge symmetry and the $\theta$-vacuum}

Even after the complete gauge fixing at the classical level, the Lagrangian (3) has a residual large gauge symmetry characterized by the gauge function, linear in $x^{-}$

$$
\Lambda_{\nu}=\frac{\pi}{e L} \nu x^{-}, \quad \nu \in Z
$$

which tends to a non-zero constant at $x^{-}= \pm L$. The linearity in $x^{-}$and the combination of constants in the coefficient are the consequence of a requirement to maintain boundary conditions for the gauge and fermi fields, respectively.

As discussed above, the bosonization rules (5) - (7) can only be applied in the gauge-fixed situation. The persistant part of the original symmetry in the bosonized LF Hamiltonian (26) consists of constant shifts of $A_{0}^{+}$[11]:

$$
A_{0}^{+} \rightarrow A_{0}^{+}-\frac{2 \pi}{e L} \nu
$$

Although $P^{-}$does not explicitly dependend on $A_{0}^{+}$, an important piece of information carried by this gauge degree of freedom is encoded in the first commutation relation in (24). The latter can be used to eliminate at the state vector level the arbitrariness related to the constant shifts (28). To do this, it is helpful to define the rescaled ZM variables [11]

$$
A_{0}^{+}=\frac{2 \pi}{e L} \hat{\zeta}, \quad \Pi_{0}^{-}=\frac{e}{2 \pi} \hat{\pi}_{0},
$$

in terms of which the basic commutation relation takes a simple quantummechanical form, independent of the box length L:

$$
\left[\hat{\zeta}, \hat{\pi}_{0}\right]=i
$$

It is simple to see that quantum-mechanically the shift transformation

$$
\hat{\zeta} \rightarrow \hat{\zeta}-\nu
$$

is realized by the unitary operator $\hat{T}_{\nu}=\left(\hat{T}_{1}\right)^{\nu}$

$$
\hat{\zeta} \rightarrow \hat{T}_{\nu} \hat{\zeta} \hat{T}_{\nu}^{\dagger}, \quad \hat{T}_{\nu}=\exp \left(-i \nu \hat{\pi}_{0}\right)
$$


The same operator transforms also the vacuum state. In the coordinate representation, $\hat{\pi}_{0}$ and the vacuum are expressed as $\left(a_{0}\right.$ is the $\mathrm{ZM}$ annihilation operator)

$$
\hat{\pi}_{0}=-i \frac{d}{d \zeta}, a_{0} \psi_{0}(\zeta) \equiv\left(\zeta+\frac{d}{d \zeta}\right) \psi_{0}(\zeta)=0, \quad \psi_{0}(\zeta)=\pi^{-\frac{1}{4}} \exp \left(-\frac{1}{2} \zeta^{2}\right) .
$$

Then the displacement operator $\hat{T}_{1}$ acts simply on the trivial vacuum $\psi_{0}(\zeta)$ :

$$
\psi_{0}(\zeta) \rightarrow \hat{T}_{\nu} \psi_{0}(\zeta)=\psi_{\nu}(\zeta)=\pi^{-\frac{1}{4}} \exp \left(-\frac{1}{2}(\zeta-\nu)^{2}\right)
$$

In this way, there are infinitely many degenerate vacuum states $\psi_{\nu}, \nu \in Z$, corresponding to the infinite set of shifted $\mathrm{ZM}$ variables $A_{0}^{+}$(or $\zeta$ ). The operator $\hat{T}_{1}$ acts as a raising operator and to have a vacuum state, invariant under $\hat{T}_{1}$, we need to superimpose all states to form the $\theta$-vacuum:

$$
|\theta\rangle=\sum_{\nu=-\infty}^{\infty} e^{-i \nu \theta} \psi_{\nu}(\zeta)|0\rangle,
$$

$\left(|0\rangle\right.$ is the vacuum with respect to the $\phi_{n}\left(x^{-}\right)$field) with the desired propery invariance up to a phase

$$
\hat{T}_{1}|\theta\rangle=e^{i \theta}|\theta\rangle
$$

Thus, that part of the original gauge symmetry, which is not related to the redundant gauge degrees of freedom, gives rise - when realized in accord with quantum mechanics - to the multiple vacua. The requirement of gauge invariance of the true physical vacuum then implies existence of the $\theta$-vacuum for the massive Schwinger model quantized on the light front. Due to bosonization, the structure of the coherent-state vacua $\psi_{\nu}(\zeta)$ is fully described by only one gauge degree of freedom and is actually very simple. The same task will be harder within the original fermion representation, because one has to find a mechanism to enrich the vacuum by a fermion component. The latter is inevitable for a non-zero chiral condensate.

In the present formulation, it is not too difficult to calculate even the $O(m)$ correction to the condensate. For this purpose, one notes that the ZM part of the scalar field in the bosonized form of the fermi field bilinear (7) is due to the definition (23) expressed as

$$
\phi_{0}=\frac{1}{\mu} \Pi_{A_{0}^{+}}-\frac{1}{\mu} \Pi_{0}^{-} .
$$

Using the rescaled momentum $\hat{\pi}_{0}$, one arrives at

$$
\bar{\psi} \psi=K: \cos \left(c \phi_{n}+c \phi_{0}\right):=K: \cos \left(c \phi_{n}+\frac{c}{\mu} \Pi_{A_{0}^{+}}-\hat{\pi}_{0}\right): .
$$

Recall however that $\Pi_{A_{0}^{+}}$is not an indpendent variable - it has to obey the secondary constraint $\chi_{2}$ Eq. (16)

$$
\Pi_{A_{0}^{+}}=\frac{m K c}{\mu}\left(: \sin \left(c \phi_{n}+c \phi_{0}\right):\right)_{0},
$$


where the argument of sin on the r.h.s. itself contains $\Pi_{A_{0}^{+}}$through $\phi_{0}$ (37). It is thus evident that we are dealing with a highly non-linear problem which can only be solved approximately. A natural method is to iterate in the fermion mass $m$. Writing cos in the exponential form, one gets

$$
: \cos c \phi:=\frac{1}{2}\left[: \exp \left(i c \phi_{n}+i \frac{c}{\mu} \Pi_{A_{0}^{+}}^{(1)}\right): \hat{T}_{1}+\text { h.c. }\right],
$$

where

$$
\Pi_{A_{0}^{+}}^{(1)}=\frac{m K c}{\mu}\left(: \sin \left(c \phi_{n}-\hat{\pi}_{0}\right):\right)_{0}
$$

is the lowest-order (in $m$ ) approximation of $\Pi_{A_{0}^{+}}$. After expanding (40) in $m$ and using the $\theta$-vacuum property (36), one readily finds

$$
\langle\theta|\bar{\psi} \psi| \theta\rangle=K\langle\theta|: \cos c \phi:| \theta\rangle=K \cos \theta-m \frac{e^{2 \gamma_{E}}}{\pi} \sin ^{2} \theta
$$

where the infinite factor $\langle\theta \mid \theta\rangle$ has been devided out. This result is rather close to that obtained in [21], where the $\theta$ - dependence of the $O(m)$ term has been found to be

$$
0.742 \sin ^{2} \theta+0.033 \cos ^{2} \theta
$$

The reason for a small discrepancy in the values of numerical coefficients is that we have been a bit careless in treating the exponential of operators. Factorization of $\cos \left(c \phi_{n}+\phi_{0}\right)$ should be done by the Baker-Campbell- Hausdorff (BCH) formula taking into account the commutator (25), whose r.h.s. is again an operator. The BCH formula thus generates a chain of commutators in the exponential representation of $\cos c \phi$ and this leads to a small correction of our result (42). The details will be given in the forthcoming publication [22]. In any case, the non-canonical commutator (25) is crucial for obtaining the correct chiral condensate to $O(m)$ in the bosonized massive light-front Schwinger model.

\section{Acknowledgments}

This work has been supported in part by the Grant No. 2/1156/94 of the Slovak Grant Agency for Science, in part by the NSF Grant No. INT-9515511 and by the U.S. Department of Energy, Grant No. DE-FG02-87ER40371.

\section{References}

[1] J. Schwinger, Phys. Rev. 128 (1962) 2425.

[2] T. Eller, H.C. Pauli, S.J. Brodsky: Phys. Rev. D35 (1987) 1493.

[3] Th. Heinzl, St. Krusche and E. Werner, Phys. Lett. B 275 (1992) 410.

[4] G. McCartor, Z. Phys. C64 (1994) 349.

[5] G. McCartor, S. Pinsky and D. Robertson, Phys. Rev. D56 (1997) 1035. 
[6] J. H. Lowenstein and J. A. Swieca, Ann. Phys. (N.Y.) 68 (1971) 172.

[7] G. Morchio, D. Pierroti and F. Strocchi, Ann. Phys. (N.Y.) 188 (1988) 217.

[8] T. Maskawa and K. Yamawaki, Prog. Theor. Phys. 56 (1976) 270.

[9] V. A. Franke, Yu. V. Novozhilov and E. V. Prokhvatilov, Lett. Math. Phys. 5 (1981) 239; Lett. Math. Phys. 5 (1981) 437.

[10] T. Heinzl, S. Krusche, E. Werner, Phys. Lett. B256 (1991) 55.

[11] A. C. Kalloniatis and D. G. Robertson, Phys. Lett. B381 (1996) 209.

[12] L. Martinovič, Phys. Lett. B400 (1997) 335.

[13] P. A. M. Dirac, Lectures on Quantum Mechanics, Academic Press, New York, 1964.

[14] E. V. Prokhvatilov, H. W. L. Naus and H. - J. Pirner, Phys. Rev. D51 (1995) 2933.

[15] J. P. Vary, T.J. Fields and H. - J. Pirner, Phys. Rev. D53 (1996) 7231.

[16] A. Harindranath and J. P. Vary, Phys. Rev. D37 (1988) 3010.

[17] P. Steinhardt, Ann. Phys. (N.Y.) 128 (1980) 425.

[18] J. Kogut and L. Susskind, Phys. Rev. D11 (1975) 3594.

[19] S. Coleman, Phys. Rev. D11 (1975) 2088.

[20] A. C. Kalloniatis and H.-C. Pauli, Z. Phys. C60 (1993) 255.

[21] C. Adam, Ann. Phys. (N.Y.) 259 (1997) 1.

[22] L. Martinovič and J. P. Vary, to be published. 